\title{
Fault Detection and Diagnosis of Air Handling Unit: A Review
}

\author{
Cheng Yew Leong \\ 1Razak Faculty of Technology and Informatics, Universiti Teknologi Malaysia, Jalan Sultan Yahya Petra, 54100 Kuala Lumpur, Malaysia
}

\begin{abstract}
Air-conditioning systems consumed the most energy usage nearly $45 \%$ of the total energy used in commercial-building. Where AHU is one of the most extensively operated equipment and this device is typical customize and complex which can results in hardwire failure and controller errors. The efficiency of the system is very much depending on the proper functioning of sensors. Faults arising from the sensors and control systems are a major contribution to the energy wastage. As such faults often go unnoticed for extended periods of time until the deterioration in performance becomes great enough to trigger comfort complaints or total equipment failure. Energy could be reduced if those faults can be detected and identified at early stage. This paper aims to review of various existing automated fault detection and diagnosis (AFDD) methods for an Air Handling Unit. The background of AHU system, general fault detection and diagnosis framework and typical faults in AHU is described. Comparison and evaluation of the various methodologies will be reviewed in this paper. This comparative study also reveals the strengths and weaknesses of the different approaches. The important role of fault diagnosis in the broader context of airconditioning is also outlined. By identifying and diagnosing faults to be repaired, these techniques can benefits building owners by reducing energy consumption, improving indoor air quality and operations and maintenance.
\end{abstract}

\section{Introduction}

Heating, Ventilating, and air-conditioning (HVAC) systems are important parts of a building system. HVAC systems provide building occupants with a comfortable and productive environment. However, HVAC systems become more and more complex to satisfy the increasing demands on indoor environment quality (IEQ) and energy consumption in modern buildings. The energy consumption of building HVAC systems constitutes 14 $\%$ of the primary energy consumption in the U.S and about $32 \%$ of the electricity generated in the U.S. [1]. It is a big challenge to improve the whole building energy efficiency while maintaining the indoor environmental quality. Building management systems (BMSs) are therefore widely used to achieve automatic monitoring and control of HVAC systems. Sensors play essential roles in BMSs. However, sensor faults may cause measurements misrepresent the operation condition of HVAC systems; mislead control systems and consequently cause energy waste, an increase in operation costs, and unacceptable IEQ. Reliable sensors are essential for reliable monitoring and control of HVAC systems.

Faults in building HVAC systems, including design problems, equipment and control system malfunction, may result in energy waste. These problems of faults include mechanical failures such as dampers or actuators stuck, leaking valves, biased sensors, in appropriate operations intervention. If early detection and diagnosis of faults are possible, energy waste could be avoided. There are two basic fault categories, degradation and abrupt faults. Degradation faults happen after sometimes of operation and gets worse gradually over time. These faults usually cannot be noticed until the degradation has exceeded a critical level. Abrupt failure faults means the equipment suddenly stops working and suffer from complete failure [2].

This paper presents a review of various AFDD methods in an AHU and mainly concerned on the overview of FDD in generic HVAC equipment.

\section{Background of Air Handling Unit}

The AHU is an important component of HVAC systems. Its main function is to control indoor air quality by supplying conditioned outdoor air. The mixed air, carefully dosed mixture of outdoor air and return air from the indoor zone, is conditioned in the AHU. The conditioning is achieved by passing the mixed air flow over the heating and/or cooling coils, as necessary. Once the air has been conditioned to the prescribed temperature and humidity levels, it is supplied to the indoor environment. Sensors play essential roles in

*tonycheng@rocketmail.com 
BMSs. However, sensor faults may cause measurements misrepresent the operation condition of HVAC systems; mislead control systems and consequently cause energy waste, an increase in operation costs, and unacceptable IEQ. The main purpose of fault detection and diagnosis (FDD) is, to detect, locate and if possible, predict the presence of the defects causing faulty operation.

There are two common types of AHU systems, are constant air volume system (CAV) and variable air volume system (VAV). The main differences between these two systems are where VAV system modulate the air flow according to the variation of a building load conditions. In VAV, the supply fan is equipped with variable frequency drive (VFD) and the air flow can be adjusted according to the load condition changes, whereas a CAV system supplies constant air flow to a conditioned zone regardless the building load is changed or not. Fig. 1 show a typical singleduct VAV system.

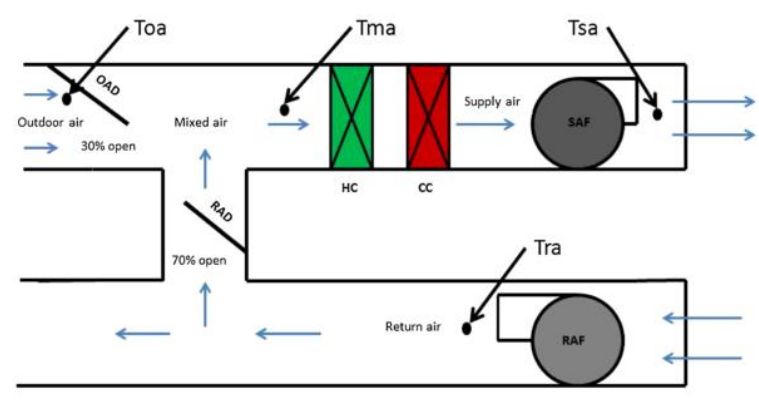

RAD=Return air damper; OAD=Outdoor air damper; SAF=Supply air fan; RAF=Return air fan

Fig 1. Air Handling Unit

Fig. 1 shows a typical AHU with air loop measurement. The control loops of a typical AHU include damper control, temperature control and pressure control. Advanced control strategies are implemented to provide adequate outdoor air ventilation and suitable supply air temperature and indoor pressure, and to minimize energy use. PID controllers are employed to control the supply air temperature, supply static pressure, outdoor air flow rate and return fan speed [3]. Optimal control strategies are used to reset the set points of the local PID control loops of supply air temperature and supply static pressure.

AHUs have four primary modes of operation during occupied periods for maintaining the supply air temperature at set point which are Heating mode, Cooling with outdoor air, Mechanical Cooling with $100 \%$ outdoor air and Mechanical cooling with minimum outdoor air. Sequencing logic determines the mode of operation of an AHU at any particular time.

There are a number of papers on neural network applications in process fault diagnosis and it is not possible to provide an exhaustive review of all the approaches here. However, the papers that we have reviewed in this part should provide the reader a good entry point to the literature in this area. If one has complete knowledge of all inputs and outputs of the system, including all forms of interactions with the environment, fault diagnosis would be a well-defined problem regardless of the number of faults present. On the other hand, if there is only a single sensor indicating whether the system is normal or faulty, then nothing can be diagnosed including the proper functioning of the sensor itself. Effectiveness of any diagnostic procedure is limited by the availability of sensor information

\section{Typical Faults in Air-Handling Unit}

\subsection{Failure of AHU Equipment}

In Fig. 2, faults occurring in $\mathrm{AHU}$ equipment can be separated into two causes. First cause is from system disturbance which normally interacts with a modelling approach such as a simplified model. The model could have sorts of uncertainties if essential terms are excluded or neglected from the plant. The uncertainties could result in uncontrollable parameters and be sensitive to system disturbance, and eventually lead to parameter failures. These failures perform as independent variables and the system controller cannot handle the uncertainties.

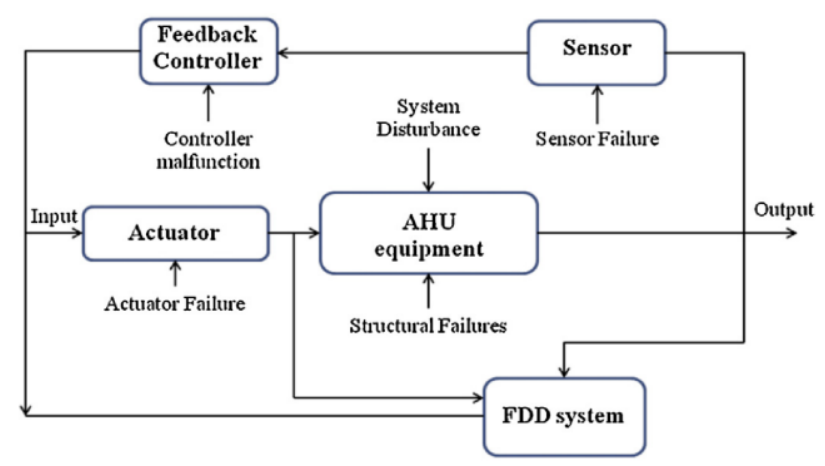

Fig 2. A general FDD framework

Second cause is structural failures that involve malfunctions in the equipment of an AHU. The structural changes lead to the variation of the process. When the equipment is in incomplete status such as air leakage in duct, failures could occur in a system in terms of insufficient heating/cooling or increasing infiltration. Other example is a dirty coil that leads to the change of heat coefficient resulting in reduced thermal conditioning capacity. Table 1 tabulates typically possible faults that occur in the equipment of an AHU resulting in the degradation of system performance. 
Table 1. Typical faults occur in AHU equipment

\begin{tabular}{|c|l|l|}
\hline Category & \multicolumn{1}{|c|}{ Device } & \multicolumn{1}{c|}{ Typical Faults } \\
\hline Equipment & Fan & $\begin{array}{l}\text { Pressure drop is increased. } \\
\text { Overall failures of supply } \\
\text { and return fans. } \\
\text { Decrease in the motor } \\
\text { efficiency. } \\
\text { Belt slippage. }\end{array}$ \\
\hline & $\begin{array}{l}\text { Duct } \\
\text { Heating coil }\end{array}$ & $\begin{array}{l}\text { Air leakage. } \\
\text { Fouling (fin and tube) leads } \\
\text { to reduce capacity. }\end{array}$ \\
\hline & Cooling Coil & $\begin{array}{l}\text { Fouling (fin and tube) leads } \\
\text { to reduce capacity }\end{array}$ \\
\hline & $\begin{array}{l}\text { Preheating } \\
\text { coil }\end{array}$ & $\begin{array}{l}\text { Fouling and reduced } \\
\text { capacity }\end{array}$ \\
\hline
\end{tabular}

\subsection{Failure in Actuator}

Actuators on devices, such as dampers and valves directly interact with an AHU for modulating the system inputs. When failures exist in actuators, these situations could cause the deviation of the system outputs beyond acceptable limits unless these failures can be detected instantly and can be solved in time. Also, faults in these instruments could practically degrade the performance of the control system since they affect the overall output with the feedback signal to the controller.

As a result, the system outputs could be changed from the expectation or design conditions. For instance, if an OA damper is stuck, the efficiency of a temperature controller could be reduced in terms of longer rise and settling time and/or waste energy by using mechanical cooling instead of free cooling from outdoor air. Other faults of actuators are shown in Table 2 .

Table 2. Typical faults occur in AHU sensors.

\begin{tabular}{|l|l|l|}
\hline Category & \multicolumn{1}{|c|}{ Device } & \multicolumn{1}{c|}{ Typical Faults } \\
\hline Actuator & $\begin{array}{l}\text { OA, RA and EA } \\
\text { dampers }\end{array}$ & $\begin{array}{l}\text { A damper is stuck or a faulty } \\
\text { position is operated. } \\
\text { Air leakage occurs at fully } \\
\text { open and closed position. }\end{array}$ \\
\hline & $\begin{array}{l}\text { Heating coil } \\
\text { (HV), cooling } \\
\text { coil (CV) and } \\
\text { preheating coil } \\
\text { valve (PV) }\end{array}$ & $\begin{array}{l}\text { A valve is stuck, broken or } \\
\text { wrong operated position. } \\
\text { Leakage occurs at fully open } \\
\text { and closed positions of the } \\
\text { valve. }\end{array}$ \\
\hline
\end{tabular}

\subsection{Failure in Sensor and feedback controller}

Sensors are used to measure state variables in a system and then send the feedback signals to the controllers maintaining the setpoints of state variables and reducing the errors between the setpoints and measured values. As a result, controller performance practically depends on sensor performance. If faults occur in sensors such as drifting and offset, the performance of controllers will gradually degrade. For example, the temperature sensor drifts about $2{ }^{\circ} \mathrm{C}$ higher than an actual value in a zone. The controller will automatically open a cooling coil valve to supply more chilled water to the cooling coil, so the room temperature will be lower than the actual value incurring energy waste. Typical faults in sensors and controllers are given in Table 3.

Table 3. Typical faults in AHU sensors and controllers.

\begin{tabular}{|c|c|c|}
\hline Category & Device & Typical faults \\
\hline Sensor & $\begin{array}{l}\text { SA, MA, OA and } \\
\text { RA } \\
\text { Temperature } \\
\text { MA, OA and RA } \\
\text { humidity } \\
\text { OA, SA and RA } \\
\text { flow rate }\end{array}$ & $\begin{array}{l}\text { Failures of a sensor are } \\
\text { offset, discrete or drift. } \\
\text { Failures of a sensor are } \\
\text { offset, discrete or drift. } \\
\text { Failures of a sensor are } \\
\text { offset, discrete or drift. }\end{array}$ \\
\hline Controller & $\begin{array}{l}\text { Motor modulation } \\
\text { Sequence of heating } \\
\text { and cooling coil } \\
\text { valve. } \\
\text { Flow difference } \\
\text { Static pressure }\end{array}$ & $\begin{array}{l}\text { Unstable response } \\
\text { Unstable response } \\
\text { Unstable response }\end{array}$ \\
\hline
\end{tabular}

\section{Types of Fault Detection and Diagnosis}

\subsection{Analytical-based method}

Analytical-based methods mainly use the difference between measured data from a field and a modelling process in terms of residuals to detect and diagnose faults. If residuals are zero, the system is regarded as fault-free.

Depending on the modelling approaches, for dynamic or steady state, the parameter estimation methods can be first-principle, gray-box or black-box. They are theoretically distinguished by the structure of residual output errors. A first-principle model is typically generated by physical principle laws governing system behavior such as mass and energy balance in terms of static and dynamic models. Although the strengths of this category can explain the dynamic behaviour of a system and perform as an accurate estimator, it is nonconvenient for real-time computation and requires a good and fast-response controller that can shortly stabilize the system performance for detecting and diagnosing sudden faults. As a result, some sudden faults cannot be detected when its response is faster than the system performance [4]. To reduce the computational cost, simplified physical models have been developed for enhancing model-based FDD and real-time HVAC control [5].

To tackle the limitations, a gray-box model has been developed for tracking the steady-state response and detecting abrupt faults. The pattern of these models combines the physical parameters referring to system characteristics with regression techniques in terms of a static model. The non-complex models can reduce computational process for remaining the system performance. There were number of researchers proposing gray box models for FDD in AHUs [6]. In contrast by using system characteristics, dynamic blackbox models use system identification techniques for 
mathematical models which cannot govern system characteristics because the estimated variables have no physical significance. However, a black-box model may be appropriate for on-line FDD when recursive identification techniques are used to continuously estimate the parameters [7].

To enhance the performance of black-box models for AFDD and real-time intelligent control, $\mathrm{Wu}$ and Sun $[[6],[8]]$ proposed physical-based parametric and multistage regression linear parametric models, respectively, for predicting room temperature in office buildings. These equations were developed by the combination of energy balance in rooms and multiple inputs and multiple outputs of autoregressive moving average (ARMAX) model, called physical-based ARMAX. Scotton [4] developed physical-based models for predicting temperature, humidity and $\mathrm{CO} 2$ concentration in rooms. These mentioned examples can enhance the performance of black-box model for describing the physical meaning of estimated parameters.

In the process of parameter estimation as illustrated in Fig. 3, the aforementioned techniques are used to compute parameter estimator block in different mathematical models for estimating parameter outputs from process inputs and measured outputs. The parameter estimator is computed by the correlation between the measured input and output variables of a process by minimizing output errors between the measured outputs and predicted outputs. Non-zero residuals from the plant and the model can be caused by faults, or the modelling uncertainties, noise from measurement and disturbance. In these situations, the parameter estimation performs as fault diagnosis or postprocessing used to diagnosing and isolating faults from noise or disturbance.

When there are change of estimated parameters is out of the selected thresholds of the parameters, these parameters show fault signature. Suitable thresholds can be selected by two major approaches: statistical testing and norm-based residual evaluation [9]. For example, observer methods are mainly based on output residuals, so they have no requirement for estimating the variation of parameters; they use input signals and the measured signals of state variables for reconstructing linear or nonlinear mathematical models. The state space equation of the linear observer will track the state outputs by using feedback errors through feedback gain (L). Structure of the full observer-based fault detection.

The selection of the threshold is similar to a parameter estimation approach. The four main types of observers are a full-order observer, unknown input observer (UIO), Kalman filter observer and non-linear observer. Since an observer mainly in state-space methods, it is useful for allowing a frequency-based design in terms of transfer function that can minimize sensitivity because of model uncertainties [10]. However, the physically-meaningful states are lost due to simplified model or linearization approach. Similar to observers but simpler to design the structure, parity relations are presented in form of algebraic equations with state-space equations. Also, it can check the consistency of system framework with the measurements. Thus, most problems regarding to FDD can be solved by linear-algebraic framework. The residual matrix of parity-based FDD is straightforward obtained by the instruction and recommendation of references [11 - 13]. By these reasons, FDD system can easily design parity space FDD framework without solid knowledge of the advanced control theory and complex mathematical algorithms.

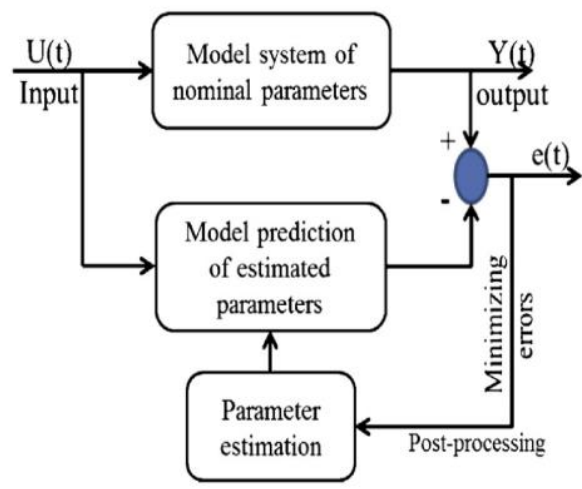

Fig 3. Schematic parameter estimation scheme

\subsection{Knowledge-based method}

For large-scale systems, if information relating to generated mathematical models are not available or are too costly and time consuming, knowledge-based methods are alternative approaches to solve these problems for fault diagnosis. These techniques are based on qualitative models that can be generally obtained through causal modeling or detailed description of systems, expert knowledge, or typical fault symptoms [14]. The three approaches in this domain have already been applied to AHU systems, namely, expert systems, pattern classification and casual analysis. For casual analysis, signed directed graph (SDG) is used for diagnosis systems since the procedures in these two methods can reflect the behaviors of systems modeled by fault symptoms without the requirement of firstprinciples. The fault decision of each node connection (node variable) can be computed by defining low and high thresholds.

One of casual analysis techniques applied to an AHU system was SDG [15]. In the second method of knowledge-based domain, the procedures can consist of an existing expert knowledge, an inference engine, or an expert system interface which can combine with the knowledge from first principles or structural description of the system in terms of rules. Meanwhile, an expert systems approach can be classified into: (1) shallowknowledge expert systems using the formulation of IFTHEN rules for generating rule-based methods; (2) deep-knowledge expert systems including functional reasoning or first-principles expert systems for diagnosing faults and (3) machine learning methods.

With the combination of shallow-knowledge (IFTHEN) and deep-knowledge expert systems (functional understandings of control system), Schein et al. [16] developed the rule set for air handling units (APAR) by 
using IF-THEN and the four mode operations of sequential control in an AHU as tabulated in Table 4, which provides the 20 rules of four modes form the total 28 rules of five modes and occupied mode operations. In each rule, a threshold value, et was computed by considering the measurement uncertainties of controlled variables and control signal from AHUs. APAR was transferred into computational algorithms embedded into BAS or AHU control as manufacturers' standard program libraries and was successfully tested in real buildings [17],[18].

Additionally, Schein and Bushby [19] developed hierarchical rule- based FDD; the results were tested and evaluated by simulation. However, expert approaches are difficult to design when knowledge acquisitions of experts and collecting of real cases are not available. A possible solution to solve this problem is to use machine learning approach, through which knowledge is automatically extracted from data by using advanced statistical theories such as hidden Markov model (HMM) and Kernel machines [20]. For an AHU application, West et al. [21] developed AFDD using statistical machine learning based on HMM; the proposed method was successfully tested in a real building. Lastly, without explicit model structures as well as analytical-based approaches, pattern classification techniques can perform non-linear correlations between data patterns and fault classes. Even though these techniques can be usefully applied to systems, where expert knowledge or certain model structures are unavailable or costly for obtaining, they essentially require robust and accurate rich data for computing potential and reliable patterns for FDD. At least four available methods of this domain applied to AHUs are (1) artificial neural networks (ANN) [7], [2224]; (2) fuzzy logic [25]; (3) support vector machine (SVM) classifier [21] and (4) Bayes classifier [26].

\subsection{Data-driven method}

Another method that also uses the relation between data patterns and fault classes in terms of modeling process is data-driven methods. These methods differ from pattern classifications since they are dimensional reduction techniques based on rigorous multivariate statistics, whereas pattern classifications learn the pattern of fault performance using entire data. According to the strength of this feature, its ability can transform high dimensional data into a lower dimension for only interested domains of data, so this approach is suitable to modern engineering systems with large-scale domains. For example, HVAC systems of medium and large commercial buildings are composed of many sensors applied to feedback control systems.

These devices possibly cause drift or off-set errors from true values resulting in the main drawback of these methods because their proficiencies depend on the quantity and quality of the process data from sensors [3]. The two main groups of data-driven methods applied to AHU systems are signal based FDD and multi-variable statistics based FDD. First one is signal processing methods consisting of: (1) periodic signals (e.g. band- pass filtering, Fourier analysis, spectral estimation and correlation functions); (2) stochastic signals; and (3) non-stationary signals which include wavelet transformation and short-time Fourier analysis [40]. However, only wavelet transformation was presently combined with PCA for fault diagnosis in AHU systems [27].

For multi-variable statistics based FDD, PCA has been extensively applied to detect faults in large systems because of the key concept that can reduce a high data volume into the useful information of a lower interested space; however, this extensive method does not use direct relation of data or tells the cause-effect relationships [28]. As a result, it is more appropriate for fault detection rather than fault diagnosis. To solve its limitation, other methods such as FDA, JAA, PLS (partial least squares), CVA (canonical variate analysis) or other pattern recognition techniques can be combined with PCA to enhance the efficient performance of fault diagnosis for on-line FDD applications in large buildings [9], [28-30].

\section{Conclusion}

This review papers presents information of FDD on AHU systems. Then, the typical faults in AHUs and the general structure of FDD for dealing with the faults investigated in the existing literature are summarized. Upon these, a classification method, which divides FDDs into three main categories, namely analyticalbased methods, knowledge-based methods, and datadriven methods, is summarized. This review also shows the strengths and weaknesses of the different diagnostic approaches. It also shows that no single method has all the desirable features for a diagnostic system. It is our view that some of these methods can complement one another resulting in better diagnostic systems.

\section{References}

[1] L. Pérez-Lombard, J. Ortiz, and C. Pout, “A review on buildings energy consumption information," Energy Build., vol. 40, no. 3, pp. 394-398, 2008.

[2] Y. Zhu, X. Jin, and Z. Du, "Fault diagnosis for sensors in air handling unit based on neural network pre-processed by wavelet and fractal," Energy Build., vol. 44, no. 1, pp. 7-16, 2012.

[3] D. Dey and B. Dong, "A probabilistic approach to diagnose faults of air handling units in buildings," Energy Build., vol. 130, pp. 177-187, 2016.

[4] S. H. Cho, H. C. Yang, M. Zaheer-Uddin, and B. C. Ahn, "Transient pattern analysis for fault detection and diagnosis of HVAC systems," Energy Convers. Manag., vol. 46, no. 18-19, pp. 3103-3116, 2005.

[5] T. . Salsbury and R. . Diamond, "Fault detection in HVAC systems using model-based feedforward control," Energy Build., vol. 33, no. 
4, pp. 403-415, 2001.

[6] S. R. Shaw, L. K. Norford, D. Luo, and S. B. Leeb, "Detection and diagnosis of HVAC faults via electrical load monitoring," ASHRAE Trans., vol. 108 PART 1, no. July, p. 468, 2002.

[7] L. K. Norford, J. A. Wright, R. A. Buswell, D. Luo, C. J. Klaassen, and A. Suby, "Demonstration of fault detection and diagnosis methods for air-handling units (ASHRAE 1020RP)," HVAC R Res., vol. 8, no. 1, pp. 41-71, 2002.

[8] S. Wu and J. Q. Sun, "Multi-stage regression linear parametric models of room temperature in office buildings," Build. Environ., vol. 56, pp. 69-77, 2012.

[9] S. Wang and F. Xiao, "Detection and diagnosis of AHU sensor faults using principal component analysis method," Energy Convers. Manag., vol. 45, no. 17, pp. 2667-2686, 2004.

[10] S. Katipamula and M. Brambley, "Methods for Fault Detection, Diagnostics, and Prognostics for Building Systems - A Review, Part II," HVAC\&R Res., vol. 11, no. 2, pp. 169-187, 2005.

[11] S. Yin, S. X. Ding, X. Xie, and H. Luo, "IEEE TRANSACTIONS ON INDUSTRIAL ELECTRONICS A Review on Basic DataDriven Approaches for," vol. 61, no. 11, pp. 110, 2014.

[12] R. Isermann, Fault-Diagnosis Systems. 2006.

[13] M. Blanke, M. Kinnaert, J. Lunze, and M. Staroswiecki, "Diagnosis and fault-tolerant control, third edition," Diagnosis Fault-Tolerant Control. Third Ed., pp. 1-695, 2016.

[14] R. Sterling, G. Provan, J. Febres, D. O'Sullivan, P. Struss, and M. M. Keane, "Model-based fault detection and diagnosis of air handling units: A comparison of methodologies," Energy Procedia, vol. 62, no. 0, pp. 686-693, 2014.

[15] J. Schein, S. T. Bushby, N. S. Castro, and J. M. House, "A rule-based fault detection method for air handling units," Energy Build., vol. 38, no. 12, pp. 1485-1492, 2006.

[16] J. Schein, S. T. Bushby, and N. S. Castro, "Results from Field Tesing of Air Handling Unit and Variable Air Volume Box Fault Detection Tools.," p. 42p, 2003.

[17] J. E. Seem, "Using intelligent data analysis to detect abnormal energy consumption in buildings," Energy Build., vol. 39, no. 1, pp. 5258, 2007.

[18] J. Schein and S.T. Bushby, "A hierarchical fault detection and diagnostic method for HVAC systems.," HVAC\&R Res., vol. 12, no. 1, pp. 111-125, 2006.

[19] S. R. West, Y. Guo, X. R. Wang, and J. Wall, "Automated fault detection and diagnosis of HVAC subsystems using statistical machine learning CSIRO Energy Technology, Newcastle , Australia CSIRO ICT Centre, Sydney, Australia."

[20] O. Morisot and D. Marchio, "Fault Detection and Diagnosis on Hvac Variable Air Volume System Using Artificial Neural Networks," ASHRAE Trans., vol. 105, p. 1087, 1999.

[21] J. Liang and R. Du, "Model-based Fault Detection and Diagnosis of HVAC systems using Support Vector Machine method," Int. J. Refrig., vol. 30, no. 6, pp. 1104-1114, 2007.

[22] W. Y. Lee, C. Park, J. M. House, and G. E. Kelly, "Fault diagnosis of an air-handling unit using artificial neural networks," ASHRAE Trans., vol. 102, no. 1, pp. 540-549, 1996.

[23] W. Y. Lee, J. M. House, and D. R. Shin, "Fault diagnosis and temperature sensor recovery for an air-handling unit," ASHRAE Transactions, vol. 103, no. 1. pp. 621-633, 1997.

[24] B. Fan, Z. Du, X. Jin, X. Yang, and Y. Guo, “A hybrid FDD strategy for local system of AHU based on artificial neural network and wavelet analysis," Build. Environ., vol. 45, no. 12, pp. 2698-2708, 2010.

[25] A. Moosavian, M. Khazaee, H. Ahmadi, M. Khazaee, and G. Najafi, "Fault diagnosis and classification of water pump using adaptive neuro-fuzzy inference system based on vibration signals," Struct. Heal. Monit., vol. 14, no. 5, pp. 402-410, 2015.

[26] V. Venkatasubramanian, R. Rengaswamy, K. Yin, and S. N. Kavuri, "A review of process fault detection and diagnosis," Comput. Chem. Eng., vol. 27, no. 3, pp. 293-311, 2003.

[27] S. Wang and J. Cui, "Sensor-fault detection, diagnosis and estimation for centrifugal chiller systems using principal-component analysis method," Appl. Energy, vol. 82, no. 3, pp. 197213, 2005.

[28] R. Dunia and S. J. Qin, "Joint diagnosis of process and sensor faults using principal component analysis," Control Eng. Pract., vol. 6, no. 4, pp. 457-469, 1998.

[29] M. Padilla and D. Choinière, "A combined passive-active sensor fault detection and isolation approach for air handling units," Energy Build., vol. 99, pp. 214-219, 2015.

[30] Y. Guo et al., "Optimized neural network-based fault diagnosis strategy for VRF system in heating mode using data mining," Appl. Therm. Eng., vol. 125, pp. 1402-1413, 2017. 